\title{
Sensitivity and spatial resolution for electron-spin-resonance detection by magnetic resonance force microscopy
}

\author{
Z. Zhanga) \\ Condensed Matter and Thermal Physics Group, Materials Science and Technology Division, MS K764, \\ Los Alamos National Laboratory, Los Alamos, New Mexico 87545 \\ M. L. Roukes \\ Condensed Matter Physics, California Institute of Technology, Pasadena, California 91125 \\ P. C. Hammel \\ Condensed Matter and Thermal Physics Group, Materials Science and Technology Division, MS K764, \\ Los Alamos National Laboratory, Los Alamos, New Mexico 87545
}

(Received 19 October 1995; accepted for publication 31 August 1996)

\begin{abstract}
The signal intensity of electron spin resonance in magnetic resonance force microscopy (MRFM) experiments employing periodic saturation of the electron spin magnetization is determined by four parameters: the rf field $H_{1}$, the modulation level of the bias field $H_{m}$, the spin relaxation time $\tau_{1}$, and the magnetic size $R(\partial H / \partial z)$ of the sample. Calculations of the MRFM spectra obtained from a 2,2-diphenyl-1-picrylhydrazyl particle have been performed for various conditions. The results are compared with experimental data and excellent agreement is found. The systematic variation of the signal intensity as a function of $H_{1}$ and $H_{m}$ provides a powerful tool to characterize the MRFM apparatus. (C) 1996 American Institute of Physics. [S0021-8979(96)00624-X]
\end{abstract}

\section{INTRODUCTION}

Recent theoretical ${ }^{1,2}$ and experimental work $^{3-9}$ has shown that magnetic resonance force microscopy (MRFM) is a new 3D imaging technique ${ }^{8,9}$ with the potential of achieving atomic scale resolution. One of the most important features of the MRFM is that it replaces the detector coil in the conventional magnetic resonance imaging (MRI) measurement with a micromechanical resonator (or microcantilever) which can sensitively detect the force between a permanent magnet and the spin moment in the sample. In the first nuclear magnetic resonance experiment using MRFM,${ }^{4}$ the reported spatial resolution $(\sim 2 \mu \mathrm{m})$ is already an order of magnitude better than that of current MRI.

Here we discuss the influence of various experimental parameters on sensitivity and spatial resolution in one class of mechanically detected magnetic resonance experiments, that is, detection of electron spin resonance (ESR) through periodic saturation of the electron spin magnetization. A key component in the MRFM setup is the magnetic tip which not only produces the necessary field gradient for the imaging, but also generates an interaction with the electron or nuclear spins in the sample. The force between the field gradient and the spin moments causes the cantilever to vibrate and its movement is monitored by an optic fiber interferometer. In order to take full advantage of the high $Q$ factor of the cantilever, the spins driven by an external rf field must be manipulated in such a way that the frequency of the time varying force matches the cantilever resonance frequency $f_{c}$

\footnotetext{
a) Also at Center for Nonlinear Studies, Los Alamos National Laboratory, Los Alamos, NM 87545. Electronic mail: zhang@rayleigh.lanl.gov
}

$(1 \sim 10 \mathrm{kHz})$. Modulation of the external field or the rf field at $f_{c}$ can lead to unacceptably large direct coupling to the cantilever. ${ }^{3}$ Two modulation techniques have been developed to avoid this problem. One involves modulation of the $\mathrm{rf}$ frequency or the external field at half the cantilever frequency (half-frequency modulation). ${ }^{3,4}$ The other involves modulation of the amplitudes of both the bias field and the $\mathrm{rf}$ field at two different frequencies (these can be anharmonic, i.e., they need not be either multiples or rational fractions of $f_{c}$ ) while keeping the sum or difference of the two frequencies at $f_{c}{ }^{5}$

Due to the complexity of these excitation schemes, it is not trivial to relate the MRFM spectra to the spin distribution within the sample - an extremely important capability for 3D imaging. In a previously reported ESR measurement using anharmonic modulation, ${ }^{6}$ despite the fact that the noise level agrees with the thermal energy analysis of the cantilever, the signal-to-noise ratio is more than an order of magnitude smaller than one estimates by assuming that all of the polarization moment in the sample is contributing at resonance. Therefore, a detailed investigation of the resonance signal as a function of various adjustable parameters (i.e., the rf field and the modulation level) is essential in order to understand the MRFM spectra. As we show, the anharmonic modulation, although very effective in reducing direct coupling, leads to a significant loss of signal intensity.

In Sec. II we calculate the ESR amplitude and the linewidth of the MRFM signal resulting from both simple field modulation at the cantilever frequency and anharmonic field modulation. In Sec. III these calculations are compared with experimental measurements which have been performed on a small particle of 2,2-diphenyl-1-picrylhydrazyl (DPPH). In Sec. IV we summarize and present conclusions. 


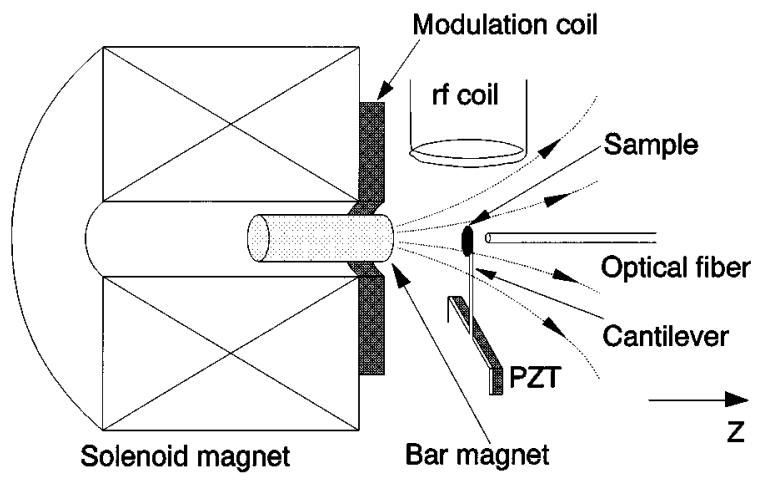

FIG. 1. A schematic diagram of the MRFM apparatus. The relevant cantilever vibration is in the $\mathbf{z}$ direction.

\section{THEORETICAL PREDICTIONS}

\section{A. Factors influencing signal magnitude}

A MRFM setup (as shown in Fig. 1) includes a bar magnet which provides the necessary field gradient $\boldsymbol{\nabla H}$ and an average magnetic field $H_{\text {bar }} \hat{\mathbf{z}}$, an electromagnet which provides a ramped bias field $H_{\text {bias }} \hat{\mathbf{z}}$, and a modulation coil which produces a field $\mathbf{H}_{m}(t)=H_{m} \sin \left(2 \pi f_{m} t\right) \hat{\mathbf{z}}$. The total magnetic field at the sample is $\mathbf{H}_{0}=\left(H_{\text {bar }}+H_{\text {bias }}+H_{m}\right) \hat{\mathbf{z}}$. Here we neglect the small field gradients perpendicular to $\hat{\mathbf{z}}$, a good approximation given the small size of the sample relative to the bar magnet. A rf field $H_{\mathrm{rf}}(t)=H_{1} e^{i \omega_{0} t}$ oriented perpendicular to $\hat{\mathbf{z}}\left(\omega_{0} / 2 \pi=500-1000 \mathrm{MHz}\right)$ is produced by a coil placed near the sample mounted on the cantilever. The spins in the sample produce a moment $\mathbf{M}$ which interacts with the field gradient producing a force on the cantilever

$$
\mathbf{F}=\mathbf{M} \cdot \boldsymbol{\nabla} \mathbf{H}=M_{z}\left(\frac{\partial H}{\partial z}\right) \hat{\mathbf{z}},
$$

where $\hat{\mathbf{z}}$ is the unit vector parallel to the bias field and/or the relevant cantilever vibration direction (since the sample and the bar magnet are coaxially aligned on the $\mathrm{z}$ axis, the field gradients along $\mathrm{x}$ and $\mathrm{y}$ axes are neglected). Because of the field gradient $\partial H / \partial z$, a shell ("sensitive slice") of constant field exists within which the magnetic resonance condition, $\omega_{0}=\gamma H_{0}$, is satisfied, where $\gamma$ is the gyromagnetic ratio. The position of this sensitive slice moves as the bias field is ramped and the width of this slice $\delta z$ is determined by the uniform resonance linewidth $\delta H_{\mathrm{lw}}$ of the sample (i.e., the resonance linewidth in a uniform field which is defined in Sec. II B below) and the field gradient: $\delta z=\delta H_{\mathrm{lw}} /(\partial H / \partial z)$. If the slice intersects the sample, saturation ${ }^{10}$ of the magnetization in this slice by the applied rf field suppresses the net magnetic moment along the $z$ direction, thus changing the force on the cantilever.

The technique of mechanical detection of magnetic resonance relies on manipulating the spin magnetization $M_{z}$ in the sample such a way that the force $F(t)=M_{z}(t) \partial H / \partial z$ varies at $f_{c}$, thus driving the mechanical resonator at its resonance frequency. Modulation of either the bias field $H_{\text {bias }}$, the rf field $H_{1}$, or the rf frequency $\omega_{0}$ will cause periodic saturation and thus a modulation of $M_{z}$. If the appropriate source is available, the simplest approach is to modulate the rf frequency $\omega_{0}$ at $f_{c} .{ }^{4}$ This is equivalent to the modulation of $H_{\text {bias }}$ at $f_{c}$ thus producing the desired timedependent driving force. In order to circumvent the spurious response that can arise with simple field (or frequency) modulation, a more complex anharmonic modulation technique $^{5}$ has also been developed which is to modulate $H_{\text {bias }}$ at $f_{m}$ and $H_{1}$ at $f_{1}$ so that $\left|f_{1} \pm f_{m}\right|=f_{c}$. Due to the nonlinear response of $M_{z}$ at resonance, a component of $M_{z}(t)$ which varies at $f_{c}$ will be generated; this produces the desired driving force.

The force is detected by measuring the oscillation amplitude of the cantilever at $f_{c}$ using an optical fiber interferometer and a lock-in amplifier. A first-order estimate of the maximum oscillation amplitude $A_{\max }$ in the MRFM measurement is ${ }^{3}$

$$
A_{\max }=F_{0} Q / k=\mathscr{L}\left(\frac{\partial H}{\partial z}\right) Q / k
$$

where $F(t)=F_{0} \sin \left(2 \pi f_{c} t\right), Q$ is the quality factor of the cantilever, $k$ is its spring constant, $\mathscr{C}=\chi_{0} H_{0} V$ is the total magnetic moment of the sample, $\chi_{0}$ is the magnetic susceptibility per unit volume, and $V$ is the total volume of the sample. However this equation assumes that the time variation of the total moment is $\mathscr{C l}_{z}(t)=\mathscr{M b} \sin \left(2 \pi f_{c} t\right)$, which is generally not the case. First, an infinite rf power would be required to fully saturate the sample at resonance (i.e., drive $M_{z}$ to zero). Second, unless the dimension of the sample is much smaller than the width of the sensitive slice, at any given moment, only a fraction of the spins in the sample is resonant at the frequency of the rf field and thus sensitive to its presence. Finally, the Fourier transform of $\mathscr{L l}(t)$ will include components at $f_{m}$ and $f_{1}$ as well as at higher harmonics of $f_{c}$. Therefore, the Fourier component at $f_{c}$ will be smaller than the total magnetic moment $\mathscr{C l}$.

In order to simplify our model calculation of the MRFM signal, a few assumptions have been made even though some of them are not absolutely necessary. First, the sample is assumed to have a spherical shape. (A Gaussian distribution of the spin density along the field gradient direction has also been calculated and the results are very similar to the calculation shown here.) Second, the sample is assumed to contain free electrons; therefore $\gamma$ is constant at $2 \pi \times 2.8 \mathrm{MHz} / \mathrm{G}$. The sample's other intrinsic parameters (i.e., susceptibility $\chi_{0}$ and spin relaxation time $\tau_{1}$ ) are chosen to have the same values as those of DPPH, ${ }^{11,12}$ a standard ESR sample. Finally, it is assumed the bias field is swept at an infinitesimal rate.

From the previous discussion, it can be seen that several factors determine the signal amplitude in the MRFM spectra. One of them is the uniform resonance linewidth $\delta H_{\text {lw }}$ of the sample which is directly related to the rf field $H_{1}$ and the spin relaxation time $\tau_{1}$ of the sample. Another is the modulation amplitude $H_{m}$ of the bias field relative to the "magnetic size" of the sample $(\partial H / \partial z) R$, where $R$ is the radius of the sample. The last factor is the strength of the rf field $H_{1}$ which not only affects the uniform resonance linewidth 


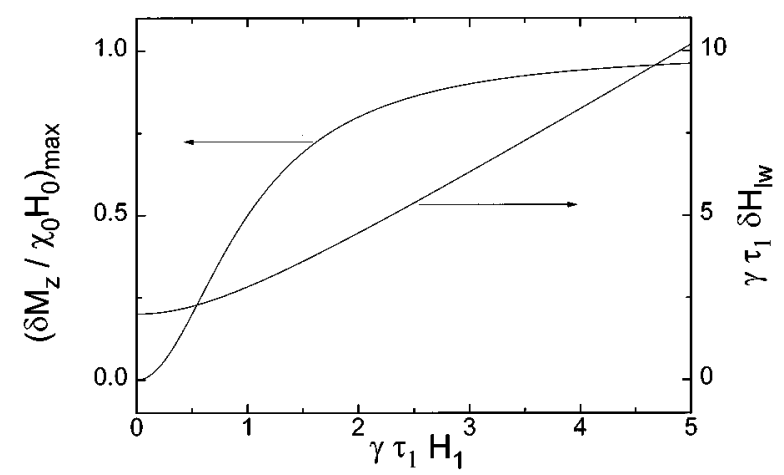

FIG. 2. The maximum value of $\delta M_{z} / \chi_{0} H_{0}$ and $\gamma \tau_{1} \delta H_{\mathrm{lw}}$ as a function of $\gamma \tau_{1} H_{1}$. The parameters used in the calculation are: $\gamma=1.76 \times 10^{7}(\mathrm{G}$ $\mathrm{s})^{-1} ; \tau_{1}=6.2 \times 10^{-8} \mathrm{~s} ; \chi_{0}=2.9 \times 10^{-6} \mathrm{~cm}^{3} / \mathrm{g}$ (from Ref. 11); and $H_{0}=295$ $\mathrm{G}$ which corresponds to the parameters of DPPH at a resonance frequency $\left(f_{0}=\gamma H_{0} / 2 \pi\right)$ of $825 \mathrm{MHz}$.

but also determines the degree of suppression of the longitudinal spin moment at resonance.

\section{B. Uniform resonance linewidth $\delta H_{\mathrm{Iw}}$ and its variation with $H_{1}$}

In the presence of an external bias field $H_{\text {bias }}$ (oriented parallel to the $z$ axis) and a transverse off field $H_{1} e^{i \omega_{0} t}$, the motion of the electron spin magnetization $\mathbf{M}$ can be derived from the Bloch equations in the rotating frame ${ }^{12}$ whose static solution gives the change of the longitudinal magnetization $\delta M_{z} \equiv \chi_{0} H_{0}-M_{z},{ }^{10}$

$$
\frac{\delta M_{z}}{\chi_{0} H_{0}}=\left(\gamma \tau_{1} H_{1}\right)^{2} \frac{1-\left(\omega_{0}-\gamma H_{0}\right) / \gamma H_{0}}{1+\tau_{1}^{2}\left(\omega_{0}-\gamma H_{0}\right)^{2}+\left(\gamma \tau_{1} H_{1}\right)^{2}} .
$$

$\delta M_{z}$ reaches a maximum value

$$
\left(\frac{\delta M_{z}}{\chi_{0} H_{0}}\right)_{\max }=\frac{H_{1}^{2}}{2 H_{0}\left(H_{r}-H_{0}\right)}
$$

at the frequency $\omega_{0}=\gamma\left(2 H_{0}-H_{r}\right)$, where $H_{r}=\sqrt{H_{0}^{2}+H_{1}^{2}+1 /\left(\gamma \tau_{1}\right)^{2}}$. Figure 2 shows the variation of $\left(\delta M_{z} / \chi_{0} H_{0}\right)_{\max }$ with the rf field $H_{1}$. When $H_{1}$ is less than $2 / \gamma \tau_{1}$, increasing the amplitude of $H_{1}$ can result in a significant enhancement of the resonance signal because this enhances the suppression of the longitudinal spin moment at resonance. This effect saturates as $H_{1}$ becomes larger than $2 / \gamma \tau_{1}$.

We define the uniform resonance linewidth $\delta H_{\mathrm{lw}}$ as the field range within which the change of the longitudinal magnetization $\delta M_{z}$ is larger than half of its maximum value $\left(\delta M_{z}\right)_{\max }$. From Eqs. (3) and (4), we obtain

$$
\delta H_{\mathrm{lw}}=2 \sqrt{\left(3 H_{r}-H_{0}\right)\left(H_{r}-H_{0}\right)} .
$$

The variation of $\delta H_{\mathrm{lw}}$ with $H_{1}$ is shown in Fig. 2. When $\gamma \tau_{1} H_{1} \geqslant 1, \delta H_{\text {lw }}$ increases almost linearly with $H_{1}$ which means more and more spins will contribute to the resonance signal as long as $\delta H_{1 \mathrm{w}} \leqslant 2 R(\partial H / \partial z)$. Therefore, in the range $\gamma \tau_{1} H_{1} \geqslant 2$, the signal intensity can still be improved with increasing $H_{1}$ because increasing the resonance linewidth causes the width of the resonance slice to widen.

\section{Bias field modulation $H_{m}$ and its relation to the MRFM spectra}

Before dealing with the more complex case of anharmonic modulation, let us consider a much simpler situation in which only the bias field is modulated at the cantilever resonance frequency $f_{c}$ (single field modulation). It is shown in the later sections that the results are qualitatively very similar between these two modulation techniques.

As mentioned before, the MRFM measures the Fourier component $A_{c}$ of the cantilever oscillation at $f_{c}$. From Eq. (2), it can be seen that

$A_{c}=2 f_{c} \int_{0}^{1 / f_{c}} d t \mathscr{L}_{z}(t) \sin \left(2 \pi f_{c} t+\phi_{0}\right)\left(\frac{\partial H}{\partial z}\right)\left(\frac{Q}{k}\right)$.

Since $A_{c}$ is maximum for $\phi_{0}=0$, we set it thus hereafter. Comparing this with Eq. (2), we can interpret the above expression to mean that only a fraction $S$ of the total moment contributes to driving the cantilever into oscillation in the MRFM experiment,

$$
S=\frac{A_{c}}{A_{\max }}=2 f_{c} \int_{0}^{1 / f_{c}} \frac{d t\left[\mathscr{\mathscr { C }} \mathrm{b}_{z}(t) \sin \left(2 \pi f_{c} t\right)\right]}{\mathscr{L} b} .
$$

The instantaneous position of the sensitive slice with respect to the center of the sample, call it $\widetilde{z}$, is determined by the magnitude of the applied field. In the absence of field modulation $\left(H_{m}=0\right)$, and in the presence of a given bias field, $\widetilde{z}$ will be a fixed value $\widetilde{z}=z_{0}$. With the addition of the modulation field $H_{m} \sin \left(2 \pi f_{c} t\right), \widetilde{z}$ acquires an oscillatory component with amplitude $z_{m}=H_{m} /(\partial H / \partial z): \widetilde{z}(t)=z_{0}$ $+z_{m} \sin \left(2 \pi f_{c} t\right)$. This will cause $\mathscr{L b}_{z}$ to become time dependent and drive the cantilever into oscillation. The scaling factor $S$ depends sensitively on the distance $z_{0}$ through $\mathscr{C l}_{z}\left(z_{0}\right)$, i.e., $S=S\left(z_{0}\right)$. Clearly $S \approx 0$ when $z_{0} \gg R$. In a bulk sample, the value of $H_{\text {bias }}$ will control the depth of the scan beneath the surface of the sample.

Replacing $\mathscr{C l}_{z}(t)$ in Eq. (7) with the integration of Eq. (3) over the particle, the scaling factor $\mathscr{S}\left(z_{0}\right)$ can be expressed as

$$
\mathscr{S}\left(z_{0}\right)=\frac{3 f_{c}}{2 R}\left(\gamma \tau_{1} H_{1}\right)^{2} \int_{0}^{1 / f_{c}} d t \sin \left(2 \pi f_{c} t\right) \int_{-R}^{R} d z^{\prime} \frac{1-\gamma(\partial H / \partial z)\left[z^{\prime}-z_{0}-z_{m} \sin \left(2 \pi f_{c} t\right)\right] / \omega_{0}}{1+\left(\gamma \tau_{1} H_{1}\right)^{2}+\left\{\gamma \tau_{1}(\partial H / \partial z)\left[z^{\prime}-z_{0}-z_{m} \sin \left(2 \pi f_{c} t\right)\right]\right\}^{2}},
$$




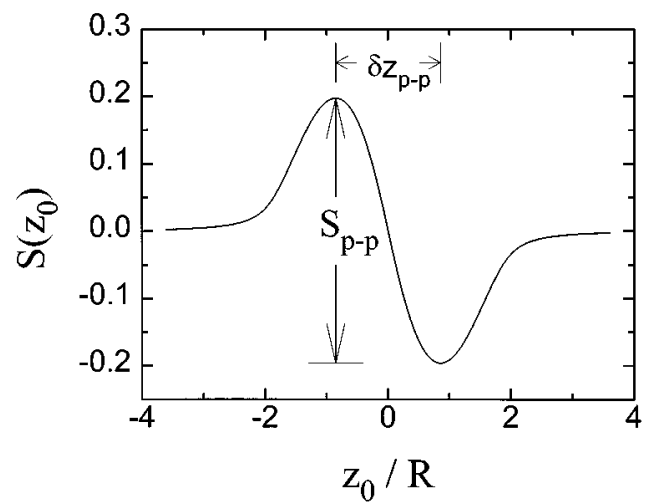

FIG. 3. The calculated MRFM spectrum (in terms of the scaling factor $S_{z}$ ) as a function of physical location of the sensitive slice using the single field modulation technique. The center of the particle is at $\mathrm{z}=0$. Parameters (taken from one set of experimental conditions) that have been used for the calculations are: $\mathrm{rf}$ frequency $f_{\mathrm{rf}}=825 \mathrm{MHz} ; \quad \gamma \tau_{1}(\partial H / \partial z) R=9.5$; $\gamma \tau_{1} H_{m}=9.3$; and $\gamma \tau_{1} H_{1}=2.1$.

where the integration over the sphere (i.e., $d z^{\prime}$ ) can be performed analytically. The result of a typical calculation of $S\left(z_{0}\right)$ as a function of $z_{0}$ is shown in Fig. 3. It is worth noting that since the time-dependent force resulting from a modulation of the position of the sensitive slice relative to the sample position is proportional to the change in force over the modulation period, a spatial gradient of the magnetization $\partial \mathscr{C l}_{z} / \partial z$ is necessary in order to have a nonzero signal.

The important parameters in Fig. 3 are the maximum change of the scaling factor $S_{\mathrm{p}-\mathrm{p}}$, which is the difference between the extrema of $S\left(z_{0}\right)$, and the spatial separation between these two extremal points which we denote $\delta z_{\mathrm{p}-\mathrm{p}}$. For a given sample, a larger $S_{\mathrm{p}-\mathrm{p}}$ means a larger resonance signal because a larger percentage of the sample is driving the cantilever into oscillation. In Fig. $4, S_{\mathrm{p}-\mathrm{p}}$ and $\delta z_{\mathrm{p}-\mathrm{p}}$ are shown as a function of the modulation length $z_{m}$ at the same value of $H_{1}$ as in Fig. 3. The existence of a maximum value $S_{\mathrm{p}-\mathrm{p} \text {,max }}$ of $S_{\mathrm{p}-\mathrm{p}}$ indicates that an optimal experimental condition is always achievable if the modulation field is properly

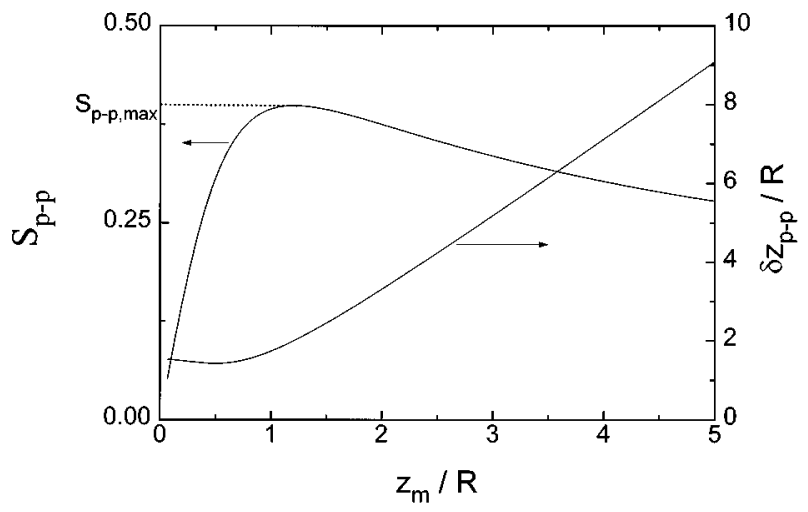

FIG. 4. The variation of the peak-to-peak scaling factor $S_{\mathrm{p}-\mathrm{p}}$ and the corresponding spatial separation $\delta z_{\mathrm{p}-\mathrm{p}} / R$ as a function of the modulation field level $z_{m} / R$. The values of $\gamma \tau_{1}(\partial H / \partial z) R, \gamma \tau_{1} H_{1}$, and the rf frequency are the same as those in Fig. 3.

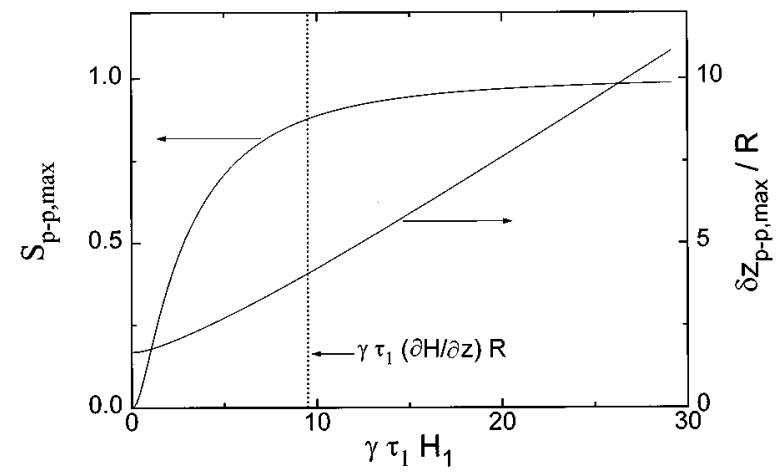

FIG. 5. The variation of the maximum value $S_{\mathrm{p}-\mathrm{p}, \max }$ of $S_{\mathrm{p}-\mathrm{p}}$ and the corresponding width of the signal described in terms of the size of the particle $\delta z_{\mathrm{p}-\mathrm{p} \text {, max }} / R$ as a function of the applied rf field $\gamma \tau_{1} H_{1}$ for $\gamma \tau_{1}(\partial H / \partial z) R=9.5$. The $\mathrm{rf}$ frequency is $825 \mathrm{MHz}$.

selected. This is also true in the conventional ESR measurement. ${ }^{13}$ The corresponding modulation field $H_{m}$ (where $S_{\mathrm{p}-\mathrm{p}}=S_{\mathrm{p}-\mathrm{p}, \max }$ ) is always close to the larger of either the magnetic size of the particle $R(\partial H / \partial z)$ or $\delta H_{\mathrm{lw}} / 2$.

When $z_{m}$ is small compared to the size of the particle $R, \delta z_{\mathrm{p}-\mathrm{p}}$ is nearly independent of $z_{m}$. The existence of a shallow minimum in Fig. 4 is directly related to the spherical shape assumption for the particle for which $\delta z_{\mathrm{p}-\mathrm{p}}=2 R$ when $z_{m}$ and $H_{1}$ approach zero. This minimum does not exist if a Gaussian distribution of the spin density is adopted. For $z_{m}>R, \delta z_{\mathrm{p}-\mathrm{p}}$ increases almost linearly with increasing $z_{m}$. Here the effective size of the particle has been spread out by the modulation field, an undesirable situation when the spatial resolution of the MRFM is a concern.

\section{D. $\mathscr{S}_{\mathrm{p}-\mathrm{p}, \max }$ and its variation with the rf field $\boldsymbol{H}_{\mathbf{1}}$}

As discussed before, increasing the rf field $H_{1}$ always results in an increase in the resonance signal, either through improved suppression of the longitudinal magnetization $\delta M_{z}$ or through the increase in the width of the resonance slice or both. This can be seen in Fig. 5 where $S_{\text {p-p,max }}$ is plotted against $H_{1}$. In this particular example, the magnetic size of the particle is chosen such that $(\partial H / \partial z) R=9.5 /\left(\gamma \tau_{1}\right)$ (the condition under which the experimental data we report below were taken), an order of magnitude larger than the intrinsic linewidth $1 / \gamma \tau_{1}$ of DPPH. The result indicates that $S_{\mathrm{p}-\mathrm{p} \text {,max }}$ increases significantly with $H_{1}$ until $H_{1} \simeq(\partial H / \partial z) R$. In the range $\gamma \tau_{1} H_{1}>10, S_{\mathrm{p}-\mathrm{p} \text {,max }}$ tends to saturate with increasing $H_{1}$, a consequence of the fact that the size of the resonance slice has become larger than the size of the particle and no more spins can be involved by increasing the uniform resonance linewidth. This is consistent with the calculation of the spatial resolution $\delta z_{\mathrm{p}-\mathrm{p} \text {,max }}\left(\right.$ when $S_{\mathrm{p}-\mathrm{p}}=S_{\mathrm{p}-\mathrm{p} \text {,max }}$ ) in Fig. 5 which shows a significant increase of $\delta z_{\mathrm{p}-\mathrm{p} \text {,max }}$ with $H_{1}$ when $\gamma \tau_{1} H_{1} \geqslant 10$.

\section{E. Minimum detectable magnetic moment}

One of the parameters of great interest is the minimum magnetic moment $\mathscr{L}_{\text {min }}$ which can be detected using the MRFM. ${ }^{14,15}$ We are now in a position to estimate $\mathscr{L}_{\text {min }}$. Figure 5 shows that, for this modulation scheme, $S_{\mathrm{p}-\mathrm{p} \text {,max }}$ 


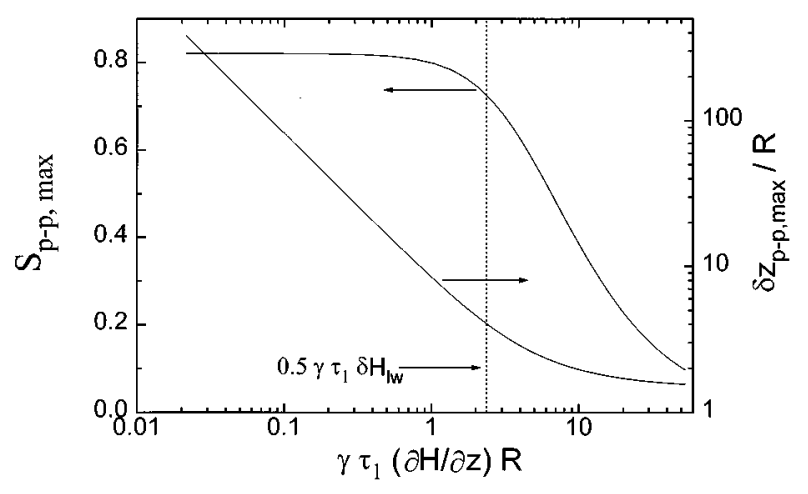

FIG. 6. The variation of the maximum value $S_{\mathrm{p}-\mathrm{p}, \max }$ of $S_{\mathrm{p}-\mathrm{p}}$ and the corresponding $\delta z_{\mathrm{p}-\mathrm{p}, \max } / R$ as a function of the magnetic size of the particle $\gamma \tau_{1}(\partial H / \partial z) R$ for $\gamma \tau_{1} H_{1}=2.1$. The rf frequency is $825 \mathrm{MHz}$.

saturates at a value of 1. From Eq. (2) and the definition of $S$ in Eq. (7), it can be seen that the maximum oscillation amplitude (i.e., half of the peak-to-peak value) of the cantilever is about $0.5 \mathscr{C}(\partial H / \partial z) Q / k$. Defining the minimum detectable oscillation amplitude as equal to the thermally driven amplitude $A_{t}$, we have $\mathscr{C}_{\min }=2 A_{t} k /[Q(\partial H / \partial z)]$.

At the value of $H_{1}$ necessary to achieve this sensitivity, the linewidth of the signal $\delta z_{\mathrm{p}-\mathrm{p} \text {,max }}$ is much larger than the size of the sample. If we wish to resolve a particle of radius $\mathrm{R}$, we reduce $H_{1}$ such that $\delta z_{\mathrm{p}-\mathrm{p} \text {,max }} \leq 2 R$. From Fig. 5 [valid for $\left.\gamma \tau_{1} R(\partial H / \partial z) \gg 1\right]$ we see that, for this situation, $S_{\mathrm{p}-\mathrm{p} \text {,max }} \simeq 0.4$ and $\mathscr{C}_{\text {min }} \simeq 5 A_{t} k /[Q(\partial H / \partial z)]$. In the case of anharmonic modulation which we discuss below, $S_{\mathrm{p}-\mathrm{p}, \max }$ is further reduced relative to this case by a factor of $\sim 5$. As a result, $\mathscr{C}_{\text {min }}$ is correspondingly increased.

\section{F. Dependence of $S_{p-p, \max }$ on the field gradient $\partial H / \partial z$}

Beside the modulation field $H_{m}$ and the rf field $H_{1}$, the other experimental parameter that can be varied in the MRFM experiment is the field gradient $\partial H / \partial z$. Increasing $\partial H / \partial z$ increases the force per spin generated on the cantilever, but it also affects the magnetic size of the particle $R(\partial H / \partial z)$. Figure 6 shows that the variation of $S_{\mathrm{p}-\mathrm{p} \text {,max }}$ with field gradient falls into regimes roughly separated by the value of gradient at which $2 R(\partial H / \partial z) \simeq \delta H_{1 \mathrm{w}}$; that is where the magnetic size of the sample becomes comparable to the width of the sensitive slice. For much smaller gradients $\left(\partial H / \partial z \ll \delta H_{1 \mathrm{w}} / 2 R\right) \quad S_{\mathrm{p}-\mathrm{p} \text {, max }}$ is nearly independent of $\partial H / \partial z$ because the total number of the spins within the sensitive slice remains constant. Since $A_{c}$ $\propto S_{\mathrm{p}-\mathrm{p}, \max } \mathscr{C l}(\partial H / \partial z) Q / k$, the cantilever oscillation amplitude increases linearly with increasing $\partial H / \partial z$. In the contrasting case where the magnetic size of the sample $2 R(\partial H / \partial z)$ is much larger than the uniform resonance linewidth $\delta H_{1 \mathrm{w}}, S_{\mathrm{p}-\mathrm{p} \text { max }}$ is inversely proportional to $\partial H / \partial z$ as shown in Fig. 6. In this regime, although the sensitivity per spin is increasing linearly with $\partial H / \partial z$, the number of spins in the sensitive slice is decreasing, so there is no net increase in the oscillation amplitude $A_{c}$. The room-temperature experiment on DPPH reported here provides an example of this. Because the sensitive slice is always narrower than the particle, the MRFM signal does not change significantly with increasing or decreasing field gradient. In order to increase the signal intensity, both the field gradient and the rf field $H_{1}$ must be raised together to an appropriate level so that the uniform resonance linewidth $\delta H_{1 \mathrm{w}}$, i.e., the slice width, is similar to or larger than $2 R(\partial H / \partial z)$.

\section{G. Anharmonic modulation}

So far we have considered the case in which only the bias field is modulated at the cantilever frequency; however, modulation techniques used in practice are more complicated. Although MRFM signals can be observed using either the half-frequency modulation ${ }^{3}$ or the anharmonic modulation method, ${ }^{5}$ only the latter is considered in this subsection. Anharmonic field modulation involves simultaneous application of a modulated $\mathrm{rf}$ field $H_{\mathrm{rf}}$ and the modulation field $H_{m}(t)$,

$$
\left|H_{1}(t)\right|^{2}=\frac{1}{2} H_{1}^{2}\left[1-\sin \left(2 \pi f_{1} t\right)\right],
$$

$$
\begin{aligned}
& H_{m}(t)=H_{m} \sin \left(2 \pi f_{m} t\right), \\
& f_{c}=\left|f_{1} \pm f_{m}\right| .
\end{aligned}
$$

From Eq. (3), the suppression of $M_{z}$ involves the product of $H_{0}(t)$ and $\left[H_{1}(t)\right]^{2}$. This multiplication leads to a mixing which produces a component at the difference frequency $f_{c}$. This component drives the oscillation of the cantilever.

The scaling factor $S(z)$ under this modulation technique is slightly different from Eq. (8),

$$
\begin{aligned}
S\left(z_{0}\right)= & \frac{3 \pi f_{c}}{R n} \int_{0}^{n /\left(2 \pi f_{c}\right)} d t\left[\gamma \tau_{1} H_{1} \sin \left(\pi f_{1} t\right)\right]^{2} \sin \left(2 \pi f_{c} t\right) \\
& \times \int_{-R}^{R} d z^{\prime} \frac{1-\gamma(\partial H / \partial z)\left[z^{\prime}-z_{0}-z_{m} \sin \left(2 \pi f_{m} t\right)\right] / \omega_{0}}{1+\left[\gamma \tau_{1} H_{1} \sin \left(\pi f_{1} t\right)\right]^{2}+\left\{\gamma \tau_{1}(\partial H / \partial z)\left[z^{\prime}-z_{0}-z_{m} \sin \left(2 \pi f_{m} t\right)\right]\right\}^{2}},
\end{aligned}
$$


where $n$ is an integer and $n /\left(2 \pi f_{c}\right)$ is the new and longer period at which $H_{1}(t), H_{m}(t)$, and the lock-in reference signal $\left(\right.$ at $f_{c}$ ) all return to their initial values. Most of the results are qualitatively similar to those previously discussed. A maximum value of $S_{\mathrm{p}-\mathrm{p}}$ always exists as a function of the field modulation level $H_{m}$. This maximum value $S_{\mathrm{p}-\mathrm{p} \text {,max }}$ increases with increasing the rf field $H_{1}$ [valid at least for $\left.H_{1}<R(\partial H / \partial z)\right]$.

At a given value of $H_{1}, S_{\mathrm{p}-\mathrm{p} \text {,max }}$ is smaller by a factor of $\sim 5$ than the calculation using the single field modulation technique. This is due to the fact that the anharmonic modulation causes $\mathscr{L}(t)$ to have Fourier components at several frequencies other than $f_{c}$ component. The linewidth $\delta z_{\mathrm{p}-\mathrm{p}, \max }$, however, does not change significantly between the two modulation cases. Therefore, the minimum moment $\mathscr{L}_{\min }$ will increase by a factor of $\sim 5$ with respect to the single field modulation technique discussed before.

\section{EXPERIMENTAL RESULTS}

We have performed an electron spin MRFM experiment on a DPPH particle using the setup shown in Fig. 1. The single crystal Si cantilever has a spring constant of $k \simeq 0.08$ $\mathrm{N} / \mathrm{m}$ and a resonance frequency of $f_{c} \simeq 15 \mathrm{kHz}$. After mounting a small DPPH sample with epoxy, $f_{c}$ reduces to $9.7 \mathrm{kHz}$ and the cantilever $Q$ factor is about 12500 in vacuum. We estimate that the total weight of the sample (DPPH + epoxy) is $\sim 13 \mathrm{ng}$.

The total bias field on the sample includes contributions from both the solenoid magnet and the bar magnet. These two contributions can be separately determined through measurement of the resonance field at various rf frequencies. The dependence of the bar magnetic field on distance from its end surface was determined by obtaining spectra at various separations between the bar magnet and the sample. The result is in excellent agreement with the theoretical prediction for a bar magnet and is used to calculate the field gradient at the position of the sample. The bias field is swept at a rate of about $2 \mathrm{G} / \mathrm{s}$ during the experiment.

In Fig. 7 we compare a typical MRFM spectrum with the previously discussed theoretical prediction [see Eq. (10)]. The rms noise is about $0.8 \AA$, close to the predicted thermal driven amplitude ${ }^{15}$

$$
A_{t}=\sqrt{2 k_{B} T Q \Delta \nu / \pi k f_{c}}=1.2 \AA,
$$

where $\Delta \nu=0.3 \mathrm{~Hz}$ is the bandwidth of the lock-in amplifier.

In the first experiment, the sample's position relative to the bar magnet is fixed, therefore, the field gradient is constant at $0.58 \mathrm{G} / \mu \mathrm{m}$. A $100 \%$ amplitude modulation is applied to the $\mathrm{rf}$ power with a modulation frequency of $f_{1}=45.67 \mathrm{kHz}$. The average rf power to the system varies from 0.1 to $1.5 \mathrm{~W}$ which corresponds to a variation of $H_{1}$ from 0.7 to $2.6 \mathrm{G}$. At each rf power, the bias field modulation level $H_{m}$ (with $f_{m}=36.98 \mathrm{kHz}$ ) was varied between 1 and $20 \mathrm{G}$. A maximum in the peak-to-peak oscillation amplitude as a function of $H_{m}$ was observed as shown in Fig. 8 (a). This maximum value is then plotted as a function of the rf field $H_{1}$ and the result is shown in Fig. 9. Treating the radius and the total weight of the DPPH particle as variable

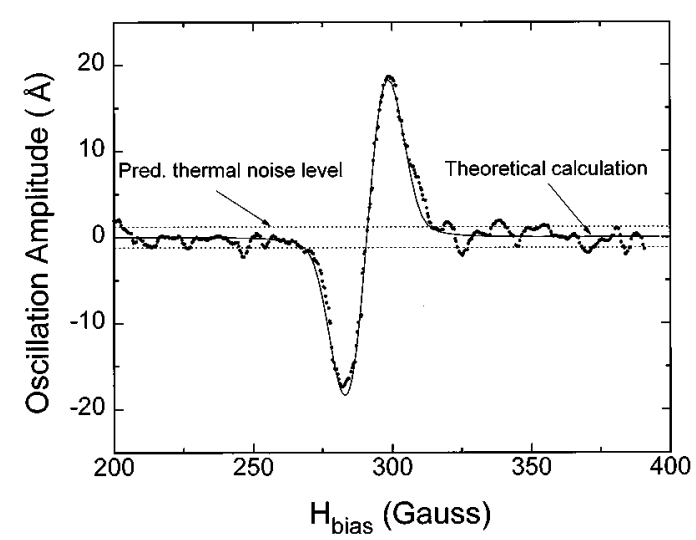

FIG. 7. An experimental MRFM spectrum of a single DPPH particle. A negative value indicates that the cantilever signal and the reference signal are out of phase. The rf frequency is $825 \mathrm{MHz}$ and the modulation depth of the rf power is $100 \%$; the modulation frequency is $45.67 \mathrm{kHz}$. The bias field is swept at a rate of $2 \mathrm{G} / \mathrm{s}$ and is also modulated at a frequency of $35.98 \mathrm{kHz}$. Other experimental conditions are $\gamma \tau_{1} H_{1}=2.1, \quad \gamma \tau_{1} H_{m}=9.3$, and $\partial H / \partial z=0.58 \mathrm{G} / \mu \mathrm{m}$. The solid line is the theoretical calculation assuming that $\gamma \tau_{1}(\partial H / \partial z) R=9.5$ (i.e., $R=15 \mu \mathrm{m}$ ), the total weight of the DPPH is $7.2 \mathrm{ng}$. A delay time of $4 \mathrm{~s}$ (or $8 \mathrm{G}$ ) has also been used. The expected thermal noise level is shown by the dotted lines.

fit parameters, both the scaling factor $S_{\mathrm{p}-\mathrm{p}}$ and the linewidth of the signal $\delta z_{\mathrm{p}-\mathrm{p}}$ can be fit by the theoretical prediction for each value of $H_{1}$. A particle radius of $15 \mu \mathrm{m}$ is obtained from this fit. This agrees with visual determination under a microscope. In addition, the total amount of DPPH from this

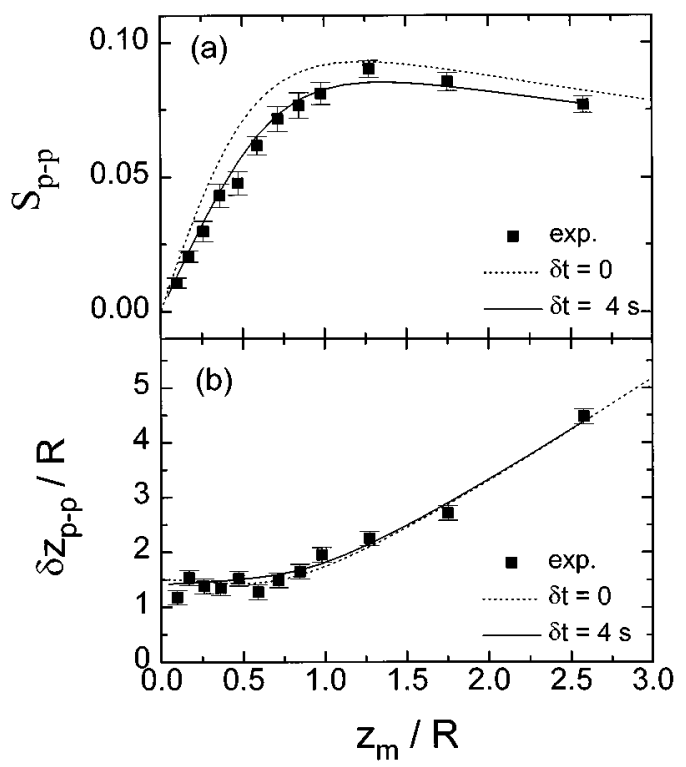

FIG. 8. (a) The peak-to-peak amplitude and (b) the linewidth (which have been converted to $S_{\mathrm{p}-\mathrm{p}}$ and $\delta z_{\mathrm{p}-\mathrm{p}} / R$ ) of the DPPH signal as a function of the modulation level $z_{m} / R$ at a constant rf field $\gamma \tau_{1} H_{1}=2.1$. The dotted lines are the calculations assuming that the field is swept at an infinitely slow rate. The solid lines are the theoretical predictions assuming that the observed signal at the time $t$ (or field $H_{\text {bias }}$ ) is the average response between $t-4 \mathrm{~s}$ (or $H_{\text {bias }}-8 \mathrm{G}$ ) and $t$ (or $H_{\text {bias }}$ ), a result of the delay response of the cantilever to the driving force. Except for the modulation level $H_{m}$, the values of other parameters used in the conversion and in the theoretical calculations are the same as those in Fig. 7. 


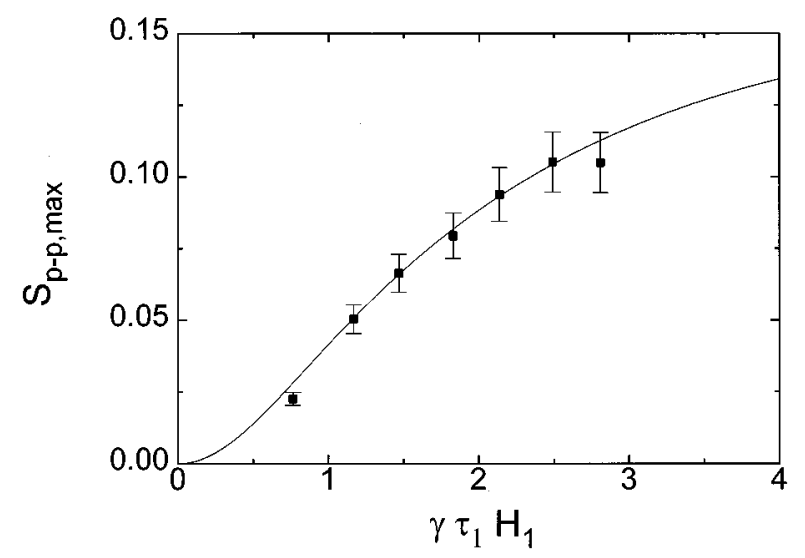

FIG. 9. The maximum value of the peak-to-peak amplitude (which has been converted to $S_{\mathrm{p}-\mathrm{p} \text { max }}$ ) of the DPPH signal as a function of the rf field $\gamma \tau_{1} H_{1}$. The solid line is the theoretical prediction using the same fitting parameters as in Fig. 7 except $H_{m}$ and $H_{1}$.

fit is $A_{0} k /\left[Q(\partial H / \partial z) \chi_{0} H_{0}\right]=7.2 \mathrm{ng}$, where $A_{0}$ is the cantilever's oscillation amplitude (in $\AA$ ) when the scaling factor $S_{\mathrm{p}-\mathrm{p}}$ is 1 . This value is also consistent with the estimate based on the frequency change of the cantilever.

In another experiment, the bar magnet is moved away from the cantilever by a distance of $0.3 \mathrm{~mm}$. As a result, the field gradient at the site of the sample changes from 0.58 to $0.21 \mathrm{G} / \mu \mathrm{m}$. This reduces the magnetic size of the sample $R(\partial H / \partial z)$ by nearly a factor of 3 . If the radius of the sample is chosen to be the same as in the previous measurement (i.e., $R=15 \mu \mathrm{m}$ ) and the total amount of DPPH $m_{\mathrm{DPPH}}$ is allowed to vary in order to get the best fit to the experimental data as shown in Fig. 10, a value of $m_{\mathrm{DPPH}}=6.7 \mathrm{ng}$ is obtained, in close agreement with the value of $7.2 \mathrm{ng}$ obtained in the previous experiment.

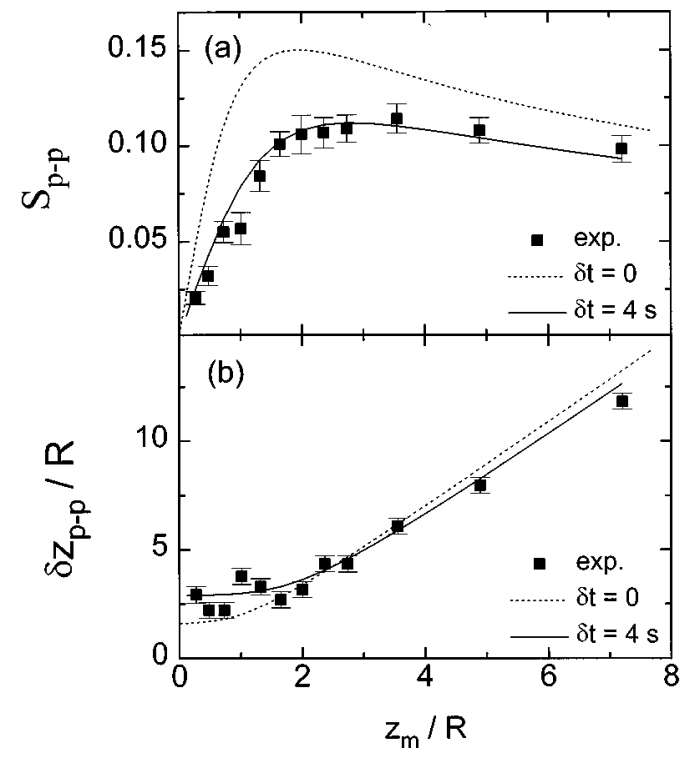

FIG. 10. The same as in Fig. 8 except that the field gradient is $0.21 \mathrm{G} /$ $\mu \mathrm{m}$ [i.e., $\gamma \tau_{1}(\partial H / \partial z) R=3.4$ ] and the total mass of DPPH is assumed to be $6.7 \mathrm{ng}$.
Figures 8(a) and 10(a) show two theoretical calculations of $S_{\mathrm{p}-\mathrm{p}}$ from Eq. (10). The dotted lines show the results obtained if it is assumed that the bias field is swept at an infinitely slow rate. However, in the real experiment the bias field is swept at $2 \mathrm{G} / \mathrm{s}$. The large $Q$ of the cantilever causes a delay $\tau_{Q}$, of order $Q / f_{c}=1.3 \mathrm{~s}$, in the response of the cantilever to a driving force. As a result, the oscillation amplitude of the cantilever at a given time $t$ (or bias field $H_{\text {bias }}$ ) is a convolution of the time-domain response function of the oscillator with the force at earlier times. Thus, the oscillation amplitude at a given time is determined by the driving force experienced for some time preceding the measurement. This time interval is characterized by the decay time of the oscillator response function which has a width $\sim \tau_{Q}$. In addition to shifting the resonance slightly (unimportant for this discussion), this will reduce the peak magnitude of the cantilever response. We have simulated the effect of this delay by replacing the oscillation amplitude at a given time $t$ (or bias field $H_{\text {bias }}$ ) by the average of the (instantaneous) response between $t-4 \mathrm{~s}$ (or $H_{\text {bias }}-8 \mathrm{G}$ ) and $t$ (or $H_{\text {bias }}$ ). The result is shown by the solid lines in Figs. 8(a) and 10(a). The $4 \mathrm{~s}$ period is chosen in order to get the best fit to the experimental data and is close to $2 Q / f_{c}$.

It is worth mentioning that the calculation of the resonance signal and its comparison with the experimental data not only gives a better understanding of the principles of the MRFM measurement, but also provides a powerful tool to characterize the experimental setup, in particular, the rf field $H_{1}$ and the modulation field $H_{m}$. Using this method, $H_{1}$ and $H_{m}$ can be determined with an uncertainty of $10 \%$ in the current system. This accuracy is better than the results obtained from other techniques we have tried (i.e., using a gaussmeter or small detective coil) which typically have an uncertainty larger than $20 \%$.

\section{CONCLUSIONS}

Due to the complexity of the MRFM experiment, the amplitude of the resonance signal depends sensitively on several intrinsic and externally adjustable parameters which include the strength of the rf field $H_{1}$, the modulation level $H_{m}$ of the bias field, the relaxation time $\tau_{1}$, and the magnetic size $(\partial H / \partial z) R$ of the sample, as follows.

(1) The relaxation time $\tau_{1}$ and the rf field $H_{1}$ determine the uniform resonance linewidth $\delta H_{\mathrm{lw}}$ of the sample which increases with increasing $H_{1}$.

(2) At a given rf field $H_{1}$ and field gradient $\partial H / \partial z$, there always exists a particular value of the modulation field $H_{m}$ at which the resonance signal reaches its maximum $S_{\mathrm{p}-\mathrm{p} \text {,max }}$. This value of $H_{m}$ can be estimated as follows:

$$
H_{m} \sim\left\{\begin{array}{ccc}
R\left(\frac{\partial H}{\partial z}\right) & \text { when } & \delta H_{1 \mathrm{w}} \ll 2 R\left(\frac{\partial H}{\partial z}\right), \\
\frac{\delta H_{1 \mathrm{w}}}{2} & \text { when } & \delta H_{1 \mathrm{w}} \gg 2 R\left(\frac{\partial H}{\partial z}\right) .
\end{array}\right.
$$

(3) $S_{\mathrm{p}-\mathrm{p} \text {,max }}$ increases with increasing $H_{1}$ either through improved suppression of the longitudinal magnetization $\delta M_{z}$ or through an increase in the width of the resonance 
slice. However, $S_{\mathrm{p}-\mathrm{p}, \max }$ tends to saturate when $\delta H_{\mathrm{lw}}$ exceeds $2 R(\partial H / \partial z) ; \delta z_{\mathrm{p}-\mathrm{p}, \max }$ increases linearly with $\delta H_{1 \mathrm{w}}$ in this region.

(4) To get the best spatial resolution, the rf field $H_{1}$ should be kept at an appropriate ratio with respect to $R(\partial H / \partial z)$ (about 0.25 if $\gamma \tau_{1} R \gg 1$ ) so that $\delta z_{\mathrm{p}-\mathrm{p}, \max } \simeq 2 R$. This requirement, however, reduces the maximum signal intensity by more than half in the limit of large $H_{1}$.

(5) The signal intensity is proportional to the field gradient $\partial H / \partial z$ only when $\delta H_{\mathrm{lw}} \gg 2 R(\partial H / \partial z)$. For $\delta H_{\mathrm{lw}} \ll 2 R(\partial H / \partial z)$, it is independent of $\partial H / \partial z$.

(6) The signal intensity can be significantly reduced by sweeping the bias field sufficiently rapidly that the sensitive slice moves through the particle in a time comparable to or less than $Q / f_{c}$.

These theoretical predictions have been verified by the experimental results on a DPPH particle. By fitting the MRFM data to the theoretical calculation, both the rf field $H_{1}$ and the modulation field $H_{m}$ have been accurately evaluated. The analysis highlights the fact that increasing $\partial H / \partial z$ does not increase the oscillation amplitude of the cantilever because the width of the signal slice is decreased. Increasing $H_{1}$ is very effective in producing large signals when $\delta H_{1 \mathrm{w}}<2 R(\partial H / \partial z)$.

\section{ACKNOWLEDGMENTS}

The authors would like to thank Dr. P.E. Wigen for helpful discussions. This work was performed under the auspices of the U.S. Department of Energy.

${ }^{1}$ J. A. Sidles , Appl. Phys. Lett. 58, 2854 (1991).

${ }^{2}$ J. A. Sidles, Phys. Rev. Lett. 68, 1124 (1992).

${ }^{3}$ D. Rugar, C. S. Yannoni, and J. A. Sidles, Nature 360, 563 (1992).

${ }^{4}$ D. Rugar et al., Science 264, 1560 (1994).

${ }^{5}$ K. J. Bruland, J. Krzystek, J. L. Garbini, and J. A. Sidles, Rev. Sci. Instrum. 66, 2853 (1994).

${ }^{6}$ P. C. Hammel, Z. Zhang, G. J. Moore, and M. L. Roukes, J. Low Temp. Phys. 101, 59 (1995).

${ }^{7}$ O. Züger, S. T. Hoen, C. S. Yannoni, and D. Rugar, J. Appl. Phys. 79, 1881 (1996)

${ }^{8}$ O. Züger and D. Rugar, Appl. Phys. Lett. 63, 2496 (1993).

${ }^{9}$ O. Züger and D. Rugar, J. Appl. Phys. 75, 6211 (1994).

${ }^{10}$ C. P. Slichter, Principles of Magnetic Resonance (Springer, New York, 1989), pp. 9, 35.

${ }^{11}$ A. V. Itterbeek and M. Labro, Physica 30, 157 (1964).

${ }^{12}$ G. Whitfield and A. G. Redfield, Phys. Rev. 106, 918 (1957).

${ }^{13}$ J. A. Weil, J. R. Bolton, and J. E. Wertz, Electron Paramagnetic Resonance (Wiley, New York, 1994), p. 500.

${ }^{14}$ J. A. Sidles, J. L. Garbini, and G. P. Drobny, Rev. Sci. Instrum. 63, 3881 (1992)

${ }^{15}$ J. A. Sidles and D. Rugar, Phys. Rev. Lett. 70, 3506 (1993). 